Artículo de posesión

\title{
Biodiversidad íctica de los mares colombianos: riqueza amenazada
}

\author{
Arturo Acero P. ${ }^{1 *}$, Andrea Polanco F. ${ }^{2}$ \\ ${ }^{1}$ Instituto de Estudios en Ciencias del Mar, Cecimar, Universidad Nacional de Colombia, Playa Salguero, Santa Marta, Colombia \\ ${ }^{2}$ Instituto de Investigaciones Marinas y Costeras, Invemar, El Rodadero, Santa Marta, Colombia \\ Artículo de posesión para el ingreso como miembro correspondiente a la \\ Academia Colombiana de Ciencias Exactas, Físicas y Naturales el 31 de mayo de 2017
}

\begin{abstract}
Resumen
Se hace un recuento de la riqueza íctica de los mares colombianos, enfatizando en los grupos amenazados de extinción en nuestras aguas y los factores de amenaza que se ciernen sobre ellos. Se detectan claras diferencias entre los peces cartilaginosos y los óseos en cuanto a sus posibilidades de supervivencia, pues mientras de los primeros 33\% (41) de las especies conocidas de Colombia se hallan amenazadas o cerca de estarlo, solo 4\% (89) de los óseos tienen esa condición. Así mismo, los niveles de conservación existentes en el Pacífico colombiano son aun claramente superiores a los del Caribe, lo cual indudablemente se asocia con la mucha mayor población humana que ocupa la costa norte colombiana. Se detallan los casos de los órdenes Carcharhiniformes (tiburones y toyos), Rhinopristiformes (peces sierra, rayas guitarras, rayas brujas), Elopiformes (sábalo) y Labriformes (peces loro) y de la familia Serranidae (meros) por considerarse ejemplos críticos que muestran la urgencia de una acción rápida, enmarcada tanto en los factores intrínsecos de amenaza de las especies, como en las necesidades de la sociedad para salvaguardar el medio circundante. (C) 2017. Acad. Colomb. Cienc. Ex. Fis. Nat.
\end{abstract}

Palabras Clave: Caribe sur; Pacífico oriental tropical; Megabiodiversidad; Ictiofauna.

Fish biodiversity of Colombian seas: threaten richness

\begin{abstract}
A review of the Colombian marine fish richness is made, emphasizing on those groups susceptible of extinction and on the factors threatening them. There are clear differences between the conservation level of Colombian bony and cartilaginous fishes; 41 species of elasmobranch fishes (33\% of total) are threatened or close to be, but only $4 \%$ ( 89 species) of the total figure of bony fishes have such status. Colombian Caribbean fishes are also more severely threatened than Pacific ones, which should be related to the higher density of human population in the northern Colombian coast. The cases of the orders Carcharhiniformes (requiem sharks), Rhinopristiformes (guitarfishes, saw fishes, witch rays), Elopiformes (tarpon) and Labriformes (parrotfishes) and the family Serranidae (groupers) are discussed in detail because they are considered critical examples showing the need of urgent action, based both on intrinsic threat factors as well as on societal needs to manage the environment. (C) 2017. Acad. Colomb. Cienc. Ex. Fis. Nat.
\end{abstract}

Key words: Southern Caribbean; Eastern Tropical Pacific; Megabiodiversity; Fish fauna.

Pisces es el nombre clásico de una de las clases en que históricamente han sido divididos los vertebrados. Sin embargo, es claro que el grupo es una construcción humana, pues sus integrantes carecen de un carácter derivado común que los defina. De ese modo, los peces son reconocidos por ser vertebrados acuáticos de sangre fría, que respiran el oxígeno disuelto en el agua por medio de branquias y que se desplazan por medio de aletas. El problema surge porque todos esos atributos tienen excepciones, es decir, existen numerosos grupos de peces que no se amoldan completamente a esa definición, ya de por sí artificial.

En cualquier caso, los taxa considerados peces superan en riqueza a los demás grupos de vertebrados sumados. Estimas recientes de la biodiversidad íctica mundial se aproximan rápidamente a 34500 especies, dado el intenso ritmo de descripción de nuevas formas, mientras que el resto de los vertebrados (no más de 32000 especies) aumentan sus números a un paso más lento (Nelson, et al., 2016; van der Laan, et al., 2017).

El estudio de los peces colombianos se inició hace poco más de un siglo con la obra del antioqueño Andrés Posada Arango (Acero P., 1988). La primera época de publicación sobre nuestra riqueza íctica marina comprende los trabajos

\footnotetext{
*Correspondencia:

Arturo Acero, aacerop@unal.edu.co

Recibido: 11 de mayo de 2017

Aceptado: 09 de junio de 2017
} 
del estadounidense Henry W. Fowler $(1944,1953)$ y del sueco George Dahl (1964, 1971). La divulgación de la tesis de maestría de Francisco J. Palacio (1974) marcó el comienzo de la nueva época de estudio de los peces marinos colombianos. Este trabajo fue seguido por Acero P., et al. (1984) y Rubio, et al. (1987), reiniciándose así un camino que sería seguido por las nuevas generaciones de ictiólogos nacionales.

Colombia, con unas 4200 especies conocidas actualmente, alberga una cifra cercana a $12 \%$ de la totalidad de la riqueza de peces mundiales. Alrededor de $62 \%$ (unas 2600 especies) de la ictiofauna colombiana puede ser considerada marina o, al menos, habitante de los ecosistemas resultantes de la mezcla de las aguas dulces y saladas. Por ser un recurso alimentario fundamental e indispensable de nuestro país, la biodiversidad íctica se encuentra enfrentada a amenazas que hacen necesaria la evaluación periódica del riesgo de extinción de especies. Los mayores procesos de amenaza que dirigen este riesgo de extinción están asociados con el hombre: sobreexplotación del recurso, deterioro o pérdida de hábitat, contaminación, introducción de especies invasoras, cambio climático y la combinación multifactorial de varios de ellos (Mace, et al., 2008). Factores intrínsecos a las especies, tanto biológicos (tasas de fecundidad, tiempos de gestación, tasas de crecimiento, estrategias reproductivas que afectan las tasas de crecimiento poblacional y productividad de la especie) como ecológicos (endemismo, patrones de distribución geográfica, preferencia de hábitat), también entran en juego haciéndolas más o menos resilientes a las amenazas. Esta revisión analiza la biodiversidad de peces marinos colombianos, enfatizando en la alarmante situación de amenaza que se cierne sobre una porción importante de ella.

Varios insumos han sido empleados durante este análisis. En primer lugar, las obras más recientes que discuten y plantean hipótesis evolutivas sobre la sistemática de los peces (Nelson, et al., 2016; van der Laan, et al., 2017; Betancur-R., et al., 2017) facilitaron la comprensión del posicionamiento sistemático de la ictiofauna colombiana. Por otro lado, información actualizada acerca del conocimiento de la riqueza íctica nacional (Rey Carrasco y Acero P., 2002; Bolaños-Cubillos, et al., 2015; Polanco Fernández, 2015; Polanco F. y Acero P., datos inéditos), permitió dar números verídicos a ese acervo biológico. Finalmente, la publicación este año de la segunda edición del Libro Rojo de los Peces Marinos Colombianos (Chasqui, et al., 2017) condujo a la vinculación del conocimiento ictiológico con el estatus de preservación de esa riqueza nacional. Las categorías de amenaza de la Unión Internacional para la Conservación de la Naturaleza (IUCN, por sus siglas en inglés) son, de mayor a menor grado de amenaza: En Peligro Crítico, En Peligro y Vulnerable. Además, la IUCN considera otras dos categorías, Casi Amenazado y Datos Deficientes; estas son denominaciones que permiten la inclusión de una especie en el libro rojo, aunque no exista unanimidad acerca de su cuadro de amenaza. Para este análisis se han considerado como amenazadas todas las especies comprendidas bajo cualquiera de las cinco categorías mencionadas e incluidas en el Libro Rojo siguiendo criterios conservadores (107); igualmente se han adicionado otras especies incluidas en las listas globales (IUCN, 2017) en las tres categorías de amenaza (23), pero que no fueron consideradas por Chasqui, et al. (2017).

Hoy en día se considera que existen ocho clases de Craniata (Nelson, et al., 2016; Betancur-R., et al., 2017), el nombre más adecuado para el suphylum que agrupa a la gran mayoría de los cordados (Figura 1). Acrania es el otro suphylum que incluye a los invertebrados considerados filogenéticamente más cercanos a los craneados, es decir cefalocordados y urocordados. Todas las clases de craneados incluyen animales pisciformes, lo cual está claramente relacionado con el origen íctico de los tetrápodos. De esas ocho clases solo cuatro han sido detectadas en Colombia (Myxini, Chondrichthyes, Actinopteri y Dipnotetrapodomorpha); el grupo que une a los peces pulmonados y a los tetrápodos no tiene representantes marinos pisciformes, mientras que las otras tres clases sí los incluyen.

Los Myxini constituyen un pequeño grupo de seres vermiformes confinados a aguas marinas, que en los mares tropicales existen solo por debajo de las plataformas continentales; son los únicos Craniata que por carecer de vértebras pueden ser considerados invertebrados. Son relativamente conocidos en el Caribe colombiano, donde se hallan representados por unas siete especies (Mok, et al., 2001; PolancoFernández y Fernholm, 2014; Polanco-Fernández, 2015), mientras que ha sido mencionada la existencia en el Pacífico colombiano de dos especies (Wisner y McMillan, 1995;

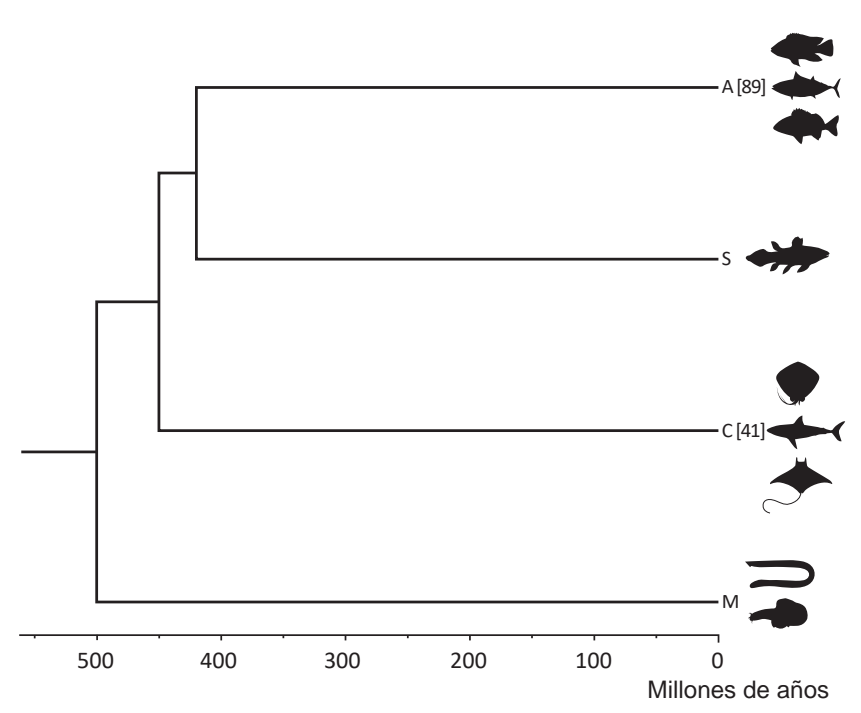

Figura 1. Filogenia de los grupos principales de cordados, modificada de Nelson, et al. (2016). Se indican el número de especies colombianas consideradas amenazadas o cerca de estarlo de peces cartilaginosos marinos y de peces óseos marinos, así como la edad de los grupos en millones de años; M Myxini, C Chondrichthyes, S Sarcopterygii, A Actinopteri. 
Rubio, et al., 2007). Se conocen factores que amenazan la super-vivencia de 19 de estos animales en otras regiones del globo como Australia, Japón y las costas meridionales del Brasil; las afectadas son especies de distribuciones restringidas, caso análogo a los myxínidos colombianos, afectadas por las prácticas destructivas de pesca y la sobreexplotación (Knapp, et al., 2011). Es necesario avanzar más en el conocimiento de nuestras especies para definir el estado de sus poblaciones.

Los peces cartilaginosos (Chondrichthyes), por su parte, son más o menos bien conocidos en los mares colombianos. Ellos pueden ser divididos en tiburones, escualos y rayas (Elasmobranchii), por un lado, y quimeras (Holocephali), por el otro. Modernamente los condrictios se agrupan en trece órdenes: uno para las quimeras (Chimaeriformes), que no incluyen especies amenazadas, y doce para el resto de peces cartilaginosos. Considerando que de aguas colombianas se conocen unas 125 especies de elasmobranquios (Navia, et al., 2016) y 41 de ellas (33\%) se hallan amenazadas o próximas a estarlo, es evidente que estos animales se encuentran cerca de ser removidos de nuestros mares, urgiéndose medidas apropiadas del Estado colombiano. Se considera que dos órdenes agrupan a la mayoría de los condrictios amenazados en Colombia: Carcharhiniformes y Rhinopristiformes. Quizá el primer factor de riesgo para la supervivencia de los peces cartilaginosos es su propia estrategia de vida; ellos dentro de sus factores intrínsecos son animales longevos, generalmente vivíparos, de crecimiento relativamente lento, con tallas de maduración sexual elevadas y, en consecuencia, bajas tasas de crecimiento poblacional; ellos desempeñan en muchas ocasiones el rol de depredadores tope en los diferentes ambientes que ocupan. El orden Carcharhiniformes, el grupo más rico de tiburones (39 especies en Colombia), incluye animales que van desde poco más de medio metro hasta tallas por arriba de cinco metros (Nelson, et al., 2016). Aparte de los rasgos mencionados arriba, estos peces son, por lo general, habitantes de las plataformas continentales que, en muchos casos, usan aguas muy someras, por ejemplo manglares, para liberar sus crías, lo cual los coloca precisamente en ambientes con alta intervención antropogénica. Es así que 19 especies (49\% de las conocidas de Colombia) del orden ameritaron su presencia en el libro rojo o se consideran amenazadas por la IUCN, 12 de ellas en la familia Carcharhinidae (BentHooker, et al., 2017b; Caldas, 2017; Caldas, et al., 2017b; Forbes, et al., 2017; Gómez, et al., 2017a, 2017b, 2017c, 2017d, 2017e; Navia, et al., 2017; Zapata, et al., 2017). Se consideran amenazadas cuatro especies de carcarínidos, tres sphírnidos y tres triákidos, mientras que otras nueve especies se encuentran muy cerca de un nivel concreto de amenaza. Igualmente, es probable que la mayoría de las especies que llegan o sobrepasan los dos metros de longitud estén amenazadas (p. ej, Carcharhinus leucas), así no hayan sido incluidas en el libro rojo. Además de los factores de preocupación ya discutidos, los tiburones son perseguidos no solo por su carne, en muchos casos de excelente calidad, sino por sus aletas, base de platos orientales, que se comercializan a precios elevados. Es por ello que la vigilancia y control efectivos en todos los frentes pueden ayudar a disminuir la enorme presión humana sobre estos peces, que no evolucionaron para enfrentarla.

Rhinopristiformes es un grupo que solo recientemente ha sido reconocido para agrupar a los peces sierra (Pristidae), a las rayas guitarra (Rhinobatidae) y a las rayas brujas (Trygonorrhinidae) (nueve especies en total en Colombia) (Last, et al., 2016a, 2016b). Las dos especies de peces sierra (Pristis pectinata y $P$. pristis) son rayas de gran tamaño (longitudes máximas por encima de $5 \mathrm{~m}$ ), armadas de una prolongación rostral equipada de grandes escamas modificadas a modo de dientes. Ellas ocurrían en aguas estuarinas y sobre fondos blandos en los mares tropicales con desembocaduras de grandes ríos, pero sus números han disminuido alarmantemente hasta su virtual desaparición de nuestras aguas (Gómez-Rodríguez, et al., 2014; Caldas y Acero P., 2017a; Caldas, et al., 2017a). La causa principal de esta enorme calamidad radica en la indiscriminada pesca de arrastre industrial y artesanal en aguas someras. La sierra y sus dientes se enredan fácilmente en las redes, lo cual conduce a su irremediable extracción. De este modo, ambas especies se encuentran críticamente amenazadas en los mares colombianos (solo P. pristis ha sido detectada en el Pacifico oriental), sin que a la fecha parezca posible diseñar una estrategia que busque la recuperación de sus poblaciones. Considerando que $P$. pristis ha sido registrada recientemente en el Pacífico chocoano (Caldas, et al., 2017a), la evaluación del número de ejemplares sobrevivientes, sus tamaños y sexos podría ser un buen comienzo. Las rayas guitarra y las brujas, por su parte, son los únicos batoideos de nuestras aguas que carecen completamente de estructuras o sistemas defensivos. Esto, sumado a sus tamaños medianos, las hacen de cierto interés para los pescadores; de este modo, dos especies de rayas guitarras y una de rayas brujas, todas de nuestro Pacífico, fueron incluidas en el libro rojo colombiano. Pseudobatos leucorhynchus es considerada vulnerable pues, aunque no presenta alto valor comercial, es afectada por la captura incidental de la pesca artesanal e industrial del camarón y su carne es vendida localmente (Payán, et al., 2011; Mejía-Falla, et al., 2017a). Pseudobatos prahli, categorizada como casi amenazada, presenta una distribución muy fragmentada y puede estar afrontando una pesquería mayor de la registrada al ser confundida con su congénere (ambas son llamadas guitarrillas) (Mejía-Falla y Navia, 2017a). Zapteryx xyster fue calificada como datos insuficientes, ya que, aunque es incidentalmente capturada en las pesquerías de camarón, tiene bajos registros y sus tendencias poblacionales se desconocen (Mejía-Falla y Navia, 2017b).

Los peces óseos (clase Actinopteri) agrupan a la mayoría de los vertebrados pisciformes, tanto a nivel global como nacional. En Colombia se conocen unas 2450 especies de peces óseos marinos, 4\% (89 especies) de las cuales se 
consideran amenazadas; la asimetría en el estado de amenaza entre peces óseos y cartilaginosos separa claramente a esos dos grupos de animales, pues los condrictios se hallan mucho más afectados por la presión humana. Al revisar la Figura 2 se nota que la amenaza, al estar dispersa a lo largo del árbol de los peces óseos, no tiene relación clara con la filogenia, exceptuando probablemente el tamaño máximo alcanzado por una especie. Se analizarán tres casos diferentes dentro de los actinopteros, en los órdenes Elopiformes, Labriformes y Perciformes. Los Elopiformes son un pequeño grupo de peces generalizados, integrado a nivel mundial por menos de diez especies, tres de las cuales existen en Colombia. La discusión se centrará en el sábalo, Megalops atlanticus (familia Megalopidae), un pez que alcanza tallas enormes, por encima de dos metros, originario del océano Atlántico (Caldas y Acero P., 2017b). Considerado muy cerca de su extirpación de nuestro Caribe, el sábalo es un pez altamente nadador que frecuenta aguas de marinas a totalmente dulces; sus registros en nuestra cuenca Caribe van desde Honda (700 km aguas arriba del río Magdalena) hasta las islas cayos del norte de la Reserva Seaflower (Caldas y Acero P., 2017b). Sus hábitos costeros lo enfrentan directamente a las actividades humanas, incluyendo sobrepesca, contaminación y destrucción del hábitat. No obstante, la principal razón de su alarmante disminución se asocia con una de las prácticas más nocivas de la zona costera: la pesca con explosivos. Esa actividad debe ser absolutamente reprimida por todos los medios del Estado dada su cercanía al terrorismo; ella extermina no solo a los peces objetivo sino a toda la vida circundante, aparte de causar graves lesiones a los pescadores. Se hace obligatorio, en este contexto, citar textualmente a George Dahl (1971, p.159) en su aparte sobre el sábalo: "Desgraciadamente, este gran recurso natural ha sido, y sigue siendo tratado de un modo estúpido y criminal. En vez de una pesca deportiva, sensata en los ríos y ciénagas (con la probabilidad del influjo del turismo) y comercial en el mar, utilizando redes agalleras de malla grande que permitan el paso de los individuos inmaduros, se está pescando a todo lo largo de la costa con dinamita. En otras palabras, cuando pasa un cardumen de sábalos, los mal llamados “pescadores” salen en sus botes y le lanzan "tacos" (cargas de dinamita). De los peces muertos y lisiados por tales descargas se recoge posiblemente un $15 \%$, pues el resto se pierde totalmente convirtiéndose en alimento para los tiburones. Por esta razón no es raro que la pesca del "Sábalo" disminuya”. En años recientes ha tenido lugar un nuevo desarrollo sobre la bioecología de la especie, su aparición en el Pacífico colombiano (Neira y Acero P., 2016). Ejemplares de sábalo empezaron a cruzar el canal de Panamá desde el Caribe hace ya más de 80 años, de modo que, a la fecha, ya se conoce de su aparición en el río Mira, frontera Colombia-Ecuador. Este evento tiene implicaciones importantes en cuanto a la conservación y manejo de esta especie y, en general, del recurso pesquero del Pacífico colombiano, pues simultaneamente está críticamente amenazada y es una especie invasora.

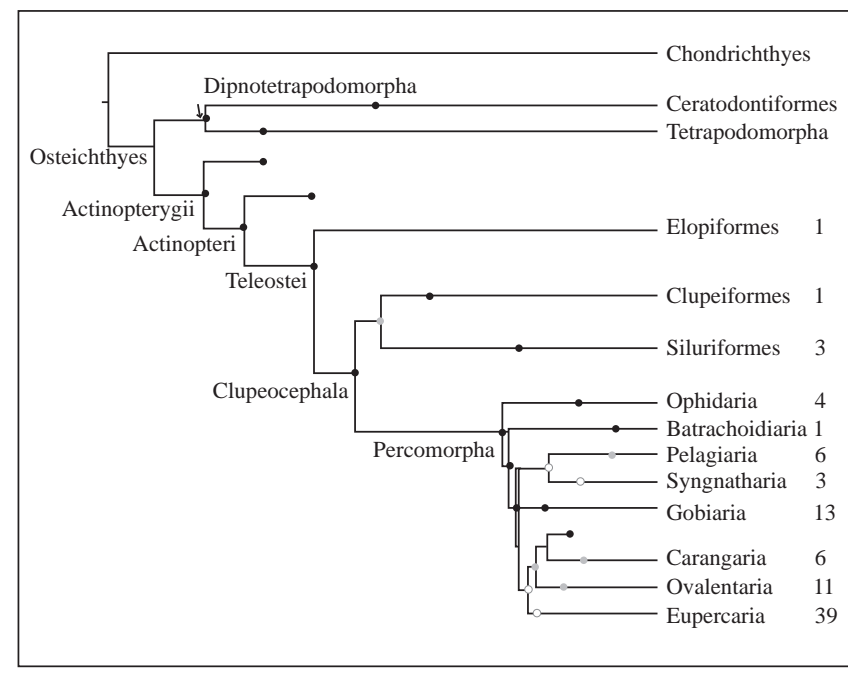

Figura 2. Filogenia de los grupos principales de Actinopteri, modificada de Betancur-R., et al. (2017). Se indican el número de especies colombianas de peces óseos marinos consideradas amenazadas o cerca de estarlo según los principales taxones que las agrupan.

El orden Labriformes incluye solo una familia en el hemisferio occidental, la de las doncellas o señoritas y sus parientes los peces loros, Labridae. Debe aclararse que aunque los peces loros sensu stricto (subfamilia Scarinae) constituyen un grupo natural claramente definido, se hallan profundamente anidados sistemáticamente dentro de los lábridos por lo que separarlos a nivel familiar implicaría una inconveniente proliferación de familias (Westneat y Alfaro, 2005). En nuestras aguas han sido registradas 44 especies, 7 (16\%) de las cuales se hallan amenazadas. De los lábridos no considerados loros hay dos especie amenazadas, el pargo pluma Lachnolaimus maximus y el lorito de Malpelo Halichoeres malpelo. El caso del pargo pluma (no un verdadero lutjánido, aunque las estadísticas pesqueras nacionales así lo indiquen) es un ejemplo agravado del caso de muchas especies arrecifales caribeñas con factores intrínsecos que actúan en contra de la resiliencia de sus poblaciones. Presenta un largo ciclo de vida, siendo hermafrodita protogínica, por lo que la captura masiva de hembras inmaduras es frecuente, en tanto que la remoción de un solo macho reduce el rendimiento reproductivo de todo un grupo (Bustos-Montes y Acero P., 2017). Los arrecifes coralinos y rocosos son cotidianamente visitados por toda clase de buzos, tanto profesionales como aficionados, que practican la pesca indiscriminada con arpones, bien sea a pulmón o con equipo autónomo. El factor agravante para el pargo pluma es que se trata de una especie de mediano tamaño e increíblemente mansa, que incluso se acerca al buceador; esto, sumado a la excelente calidad de su carne, tiene a la especie en peligro. La captura del pargo pluma con arpón, así como su comercialización, deben ser actividades vedadas, sin importar las circunstancias. El lorito de Malpelo es, por el contrario, una especie pequeña; su 
principal factor de amenaza es el endemismo, pues solo se conoce de Malpelo, la única isla oceánica colombiana del Pacífico Oriental, la cual se halla expuesta a fenómenos oceánicos abruptos consecuencia del cambio climático que podrían afectar negativamente su población (Zapata y Chasqui V., 2017).

La situación de los scarinos guarda alguna semejanza con la del pargo pluma, aunque esas especies no alcanzan el mismo nivel de aprecio. Los peces loro que crecen hasta tallas más grandes, pudiendo sobrepasar al metro de longitud (Scarus coelestinus, S. coeruleus y S. guacamaia), han sido erradicados de la gran mayoría de los arrecifes coralinos del Caribe colombiano (Bolaños-Cubillos, et al., 2017a, 2017b, 2017c, 2017d, 2017e). Esto es una calamidad enorme si se considera que son especies claves, encargadas de mantener a raya a las macroalgas que, aprovechando el cambio climático, han proliferado, cubriendo y matando a los corales por sombrío en muchos casos; igualmente, hallazgos recientes indican que los loros diseminan en sus heces a las zooxantelas, simbiontes indispensables para el crecimiento y la salud arrecifal (Castro-Sanguino y Sánchez, 2012). La situación de la más grande de estas tres especies $(S$. guacamaia) se agrava al considerar que su reclutamiento ocurre principalmente en lagunas de manglar, las cuales se hallan bajo amenaza de degradación ambiental y desaparición en razón de los desarrollos costeros fuera de control. Aparte de las tres grandes especies ya mencionadas, existen otros dos peces loros caribeños que fueron catalogados este año como casi amenazados: Scarus vetula y Sparisoma viride (Bolaños-Cubillos, 2017d, 2017e). Esas especies, de talla mediana (máximo $65 \mathrm{~cm}$ ), están siendo extraídas sin ningún control por los arponeros, al igual que por la pesca con nasas. De no tomarse medidas inmediatas es posible predecir la extirpación de estos loros en pocos años, lo cual sin duda será el golpe de gracia a nuestros ya degradados arrecifes coralinos. Solo se vislumbra como solución la eliminación total de la pesca no cientifica con arpones en los arrecifes y la prohibición de la comercialización de los scarinos.

Dentro del recientemente restringido orden Perciformes se sitúa la familia Serranidae, que incluye a peces tan estimados comercialmente como los meros. Se trata de una familia de especies hermafroditas protogínicas, en el caso de la subfamilia Epinephelinae (meros), o simultáneas, como los Serraninae. Es así que 18 de 34 (53\%) especies de epinefélinos colombianos se encuentran en alguna categoría de amenaza o cerca de ella. La situación del mero guasa (Epinephelus itajara) y del mero criollo (E. striatus), especies caribeñas críticamente amenazadas, es particularmente alarmante. En el caso del primero coinciden numerosos factores de amenaza, que van de la sobrepesca con toda clase de artes, lícitos e ilícitos, hasta la destrucción de su hábitat de reclutamiento (manglares); la captura de ejemplares de mediano tamaño agrava la situación, pues la primera madurez ocurre a tallas de poco menos de un metro (Polanco F., et al., 2017), lo cual se asemeja a lo que sucede con el sábalo. La incidencia definitiva de la sobrepesca se nota con claridad en el caso del mero criollo (Bent-Hooker, et al., 2017a). Por ejemplo, todavía en la década de los años 80 del siglo pasado la especie era frecuente y abundante en la Reserva de la Biosfera Seaflower (Garzón y Acero P., 1983; Acero P. y Garzón-Ferreira, 1991). Hoy en día el mero criollo ha sido prácticamente extirpado de las islas que conforman el departamento archipiélago (Acero P., datos inéditos). En este contexto de depauperación de la fauna de epinefélinos, la presencia en el Parque Nacional Natural Corales de Profundidad del mero negro (Hyporthodus nigritus) (Polanco F. y Acero P., 2017a), una especie críticamente amenazada en todo su ámbito de distribución en el océano Atlántico, es una noticia estimulante que debe aportar a defender la adecuada conservación de ese ecosistema. Por otro lado, el único serranino incluido en el libro rojo es Hypoplectrus providencianus, cuya distribución abarca totalmente a la Reserva de Biosfera Seaflower. Sin embargo, dado que la especie aparece en otras localidades caribeñas, se le consideró solo como casi amenazada (Polanco F. y Acero P., 2017b).

El anexo 1 lista las especies de peces marinos colombianos consideradas amenazadas (o cerca de estarlo), indicando los factores que más inciden en ese diagnóstico. La observación detenida de dicho cuadro ratifica que la principal amenaza que afronta nuestra riqueza íctica es la pesca indiscriminada, empleando incluso métodos en extremo dañinos. Es posible, así mismo, corroborar que la pesca carente de regulación y control perjudica mucho más notoriamente a los peces cartilaginosos que a los óseos. Es así que prácticamente para todos los elasmobranquios incluidos en el libro rojo colombiano (34 de 35 especies) se considera que la pesca es su principal amenaza. En el caso de los peces óseos, la amenaza proveniente de la actividad pesquera parece menos determinante, pues se conceptuó que de 74 especies de actinopteros, 69\% padece esa problemática. Para los peces óseos, en promedio más pequeños que los cartilaginosos, se consideró que la destrucción del hábitat incide en 51\% de las especies amenazadas; este factor es mencionado en solo 36\% de los elasmobranchios amenazados. La contaminación ambiental es vista como un peligro potencial para 14 especies que ocupan ambientes estuarinos durante parte o la totalidad de su ciclo vital. Fenómenos de origen global, como el El Niño Oscilación del Sur y en general el cambio climático, pueden acarrear daño a especies arrecifales o extremadamente restringidas. El endemismo es otra circunstancia a tener cuenta; es así que diez especies endémicas de nuestros mares se hallan amenazadas (Figura 3). Es imperioso que el Estado colombiano mire nuestro acervo marino y pesquero con otros ojos; que en lugar de una sucesión de entidades burocráticas inoperantes, exista por fin una política integral que vele realmente por nuestra riqueza biológica y por la seguridad alimentaria de los colombianos, claramente amenazadas hoy en día. 


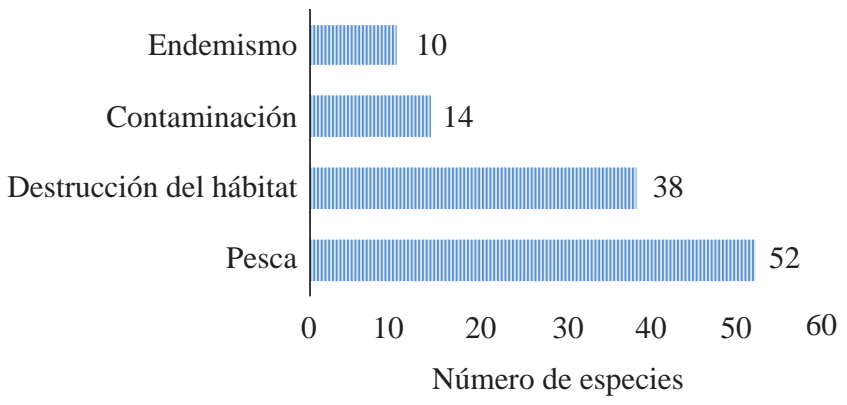

Figura 3. Principales factores que amenazan a las especies de peces marinos colombianos.

Un aspecto geográfico interesante es que los peces caribeños están mucho más amenazados que los del Pacífico americano. Descontando las 19 especies presentes en ambas costas (anfiamericanas o circuntropicales), 80 especies (62\%) de peces amenazados colombianos son caribeños, mientras que menos de la mitad (31) son exclusivos del Pacífico. El área de las aguas territoriales colombianas en el Caribe es algo más de 1.7 veces el de las aguas nacionales en el Pacífico. El estado de mayor amenaza de la ictiofauna caribeña, por encima del doble en número de especies, está seguramente relacionado con la marcada diferencia en habitantes humanos y desarrollo entre las dos regiones. Puede tratarse, en todo caso, de una combinación en el Pacífico de menor presión antrópica directa y menor posibilidad de difusión de información fidedigna sobre el estatus de las poblaciones ícticas.

Aparte de los ya mencionados factores de amenaza que se ciernen sobre la ictiofauna marina colombiana, desde hace casi una década nuestras aguas caribeñas han sido invadidas por una presencia altamente nociva, el scorpénido originario del Indo-Pacífico conocido como Pterois volitans o simplemente como pez león (González, et al., 2009; Betancur-R., et al., 2011; González-Corredor, et al., 2016). Este voraz depredador combina numerosas características y estrategias que, dado su carácter novedoso en el Atlántico occidental tropical, le han permitido proliferar de manera alarmante. Las consecuencias de su accionar en nuestros ecosistemas someros, ya de por sí en avanzado estado de deterioro, aún no han sido bien estimadas en su totalidad, pero es claro que puede coadyuvar a que peces óseos ya bajo amenaza y que recluten en los ecosistemas donde el invasor prolifera reciban un golpe definitivo. Por otro lado, dada la naturaleza oportunista del pez león, su accionar depredador se centra con frecuencia en especies pequeñas de peces. Es así que peces comunes en sitios arrecifales someros, tales como Thalassoma bifasciatum, Gramma loreto o Stegastes partitus, son algunas de las presas más importantes del invasor (Acero $\mathrm{P}$, datos inéditos). Interesantemente, el más reciente informe de la IUCN sobre los peces amenazados incluye como vulnerables a ocho especies del género Coryphopterus (Linardich, et al., 2017), pequeños gobis comunes en los fondos someros, y una de las presas favoritas del depredador invasor.

\section{Agradecimientos}

A nuestras instituciones, Universidad Nacional de Colombia sede Caribe e Instituto de Investigaciones Marinas y Costeras, por permitirnos discutir y trasmitir nuestras inquietudes. A todo el grupo de trabajo del libro rojo de peces marinos colombianos por horas de fructífera discusión sobre nuestra riqueza íctica, amenazada o no. A Maija Karala por el esquema de Latimeria. Contribución No. 456 del Instituto de Estudios en Ciencias del Mar y No. 1159 del Invemar.

\section{Conflicto de intereses}

Los autores declaran no tener conflicto de intereses.

\section{Referencias}

Acero P., A. 1988. Andrés Posada Arango, pionero de la ictiología en Colombia. Actual. Biol., 17 (63): 49-54.

Acero P., A. y J. Garzón-Ferreira. 1991. Meros, chernas y cabrillas del Caribe colombiano (Pisces: Serranidae: Epinephelinae: Epinephelini). Caldasia, 16 (78): 355-376.

Acero P., A., J. Garzón F. y F. Köster. 1984. Lista de los peces óseos conocidos de los arrecifes del Caribe colombiano, incluyendo 31 nuevos registros y descripciones. Caldasia, 14 (66): 37-84.

Acero P., A., D. Bustos-Montes, P. Pabón y A. Sanjuan. En prensa. Feeding habits of Pterois volitans: A real threat to Caribbean coral reef biodiversity. En: Makowski, C. (Ed.) Impacts of invasive species on coastal environments: Coasts in crisis. Springer.

Bent-Hooker, H., A. Rojas Archbold, N. Bolaños-Cubillos, A. Polanco F. y A. Acero P. 2017a. Epinephelus striatus (Bloch, 1792): 65-68. En: Chasqui V., L., A. Polanco F., A. Acero P., P.A. Mejía-Falla, A.F. Navia, L.A. Zapata y J.P. Caldas (Eds.). 2017. Libro rojo de peces marinos de Colombia. Serie Publicaciones Generales Invemar 93, Santa Marta. 552 p.

Bent-Hooker, H., C. Ballesteros y J.P. Caldas. 2017b. Galeocerdo cuvier (Peron \& Lesueur, 1822): 274-277. En: Chasqui V., L., A. Polanco F., A. Acero P., P.A. Mejía-Falla, A.F. Navia, L.A. Zapata y J.P. Caldas (Eds.). 2017. Libro rojo de peces marinos de Colombia. Serie Publicaciones Generales Invemar 93, Santa Marta. 552 p.

Betancur-R., R., A. Hines, A. Acero P., G. Orti, A.E. Wilbur y D.W. Freshwater. 2011. Reconstructing the lionfish invasion: insights into Greater Caribbean biogeography. J. Biogeogr., 38: 1281-1293.

Betancur-R., R., E. O. Wiley, G. Arratia, A. Acero P., M. Miya, G. Lecointre y G. Ortí. 2017. Phylogenetic classification of bony fishes. BMC Evolution. Biol.,

Bolaños-Cubillos, N., A. Abril-Howard, H. Bent-Hooker, J.P. Caldas y A. Acero P. 2015. Lista de peces conocidos del archipiélago de San Andrés, Providencia y Santa Catalina, Reserva de Biosfera Seaflower, Caribe occidental colombiano. Bol. Invest. Mar. Cost., 44 (1): 127-162.

Bolaños-Cubillos, N., A. Acero P., A. Rojas Archbold, H. BentHooker, A. Polanco F. y J.D. González. 2017a. Scarus coelestinus: 81-85. En: Chasqui V., L., A. Polanco F., A. Acero P., P.A. Mejía-Falla, A.F. Navia, L.A. Zapata y J.P. Caldas (Eds.). 2017. Libro rojo de peces marinos de Colombia. Serie Publicaciones Generales Invemar 93, Santa Marta. 552 p. 
Bolaños-Cubillos, N., A. Acero P., A. Rojas Archbold, H. BentHooker, A. Polanco F. y J.D. González. 2017b. Scarus coeruleus: 86-90. En: Chasqui V., L., A. Polanco F., A. Acero P., P.A. Mejía-Falla, A.F. Navia, L.A. Zapata y J.P. Caldas (Eds.). 2017. Libro rojo de peces marinos de Colombia. Serie Publicaciones Generales Invemar 93, Santa Marta. 552 p.

Bolaños-Cubillos, N., A. Acero P., A. Rojas Archbold, H. BentHooker, A. Polanco F. y J.D. González. 2017c. Scarus guacamaia: 91-95. En: Chasqui V., L., A. Polanco F., A. Acero P., P.A. Mejía-Falla, A.F. Navia, L.A. Zapata y J.P. Caldas (Eds.). 2017. Libro rojo de peces marinos de Colombia. Serie Publicaciones Generales Invemar 93, Santa Marta. 552 p.

Bolaños-Cubillos, N., A. Acero P., A. Rojas Archbold, H. BentHooker, A. Polanco F. y J.D. González. 2017d. Scarus vetula (Bonnaterre, 1788): 328-332. En: Chasqui V., L., A. Polanco F., A. Acero P., P.A. Mejía-Falla, A.F. Navia, L.A. Zapata y J.P. Caldas (Eds.). 2017. Libro rojo de peces marinos de Colombia. Serie Publicaciones Generales Invemar 93, Santa Marta. 552 p.

Bolaños-Cubillos, N., A. Acero P., A. Rojas Archbold, H. BentHooker, A. Polanco F. y J.D. González. 2017e. Sparisoma viride (Bloch \& Schneider, 1801): 333-337. En: Chasqui V., L., A. Polanco F., A. Acero P., P.A. Mejía-Falla, A.F. Navia, L.A. Zapata y J.P. Caldas (Eds.). 2017. Libro rojo de peces marinos de Colombia. Serie Publicaciones Generales Invemar 93, Santa Marta. 552 p.

Bustos-Montes, D. y A. Acero P. 2017. Lachnolaimus maximus (Walbaum, 1792): 77-80. En: Chasqui V., L., A. Polanco F., A. Acero P., P.A. Mejía-Falla, A.F. Navia, L.A. Zapata y J.P. Caldas (Eds.). 2017. Libro rojo de peces marinos de Colombia. Serie Publicaciones Generales Invemar 93, Santa Marta. 552 p.

Caldas, J.P. 2017. Carcharhinus longimanus (Poey, 1861): 131134. En: Chasqui V., L., A. Polanco F., A. Acero P., P.A. Mejía-Falla, A.F. Navia, L.A. Zapata y J.P. Caldas (Eds.). 2017. Libro rojo de peces marinos de Colombia. Serie Publicaciones Generales Invemar 93, Santa Marta. 552 p.

Caldas, J.P. y A. Acero P. 2017a. Pristis pectinata Latham, 1794: 44-47. En: Chasqui V., L., A. Polanco F., A. Acero P., P.A. Mejía-Falla, A.F. Navia, L.A. Zapata y J.P. Caldas (Eds.). 2017. Libro rojo de peces marinos de Colombia. Serie Publicaciones Generales Invemar 93, Santa Marta. 552 p.

Caldas, J.P. y A. Acero P. 2017b. Megalops atlanticus Valenciennes, 1847: 53-47. En: Chasqui V., L., A. Polanco F., A. Acero P., P.A. Mejía-Falla, A.F. Navia, L.A. Zapata y J.P. Caldas (Eds.). 2017. Libro rojo de peces marinos de Colombia. Serie Publicaciones Generales Invemar 93, Santa Marta. 552 p.

Caldas, J.P., P.A. Mejía-Falla, A.F. Navia y A. Acero P. 2017a. Pristis pristis (Linnaeus, 1758): 48-52. En: Chasqui V., L., A. Polanco F., A. Acero P., P.A. Mejía-Falla, A.F. Navia, L.A. Zapata y J.P. Caldas (Eds.). 2017. Libro rojo de peces marinos de Colombia. Serie Publicaciones Generales Invemar 93, Santa Marta. 552 p.

Caldas, J.P., P.A. Mejía-Falla y A.F. Navia. 2017b. Prionace glauca (Linnaeus, 1758): 281-284. En: Chasqui V., L., A. Polanco F., A. Acero P., P.A. Mejía-Falla, A.F. Navia, L.A. Zapata y J.P. Caldas (Eds.). 2017. Libro rojo de peces marinos de Colombia. Serie Publicaciones Generales Invemar 93, Santa Marta. 552 p.
Castro-Sanguino, C. y J. A. Sánchez. 2012. Dispersal of Symbiodinium by the stoplight parrotfish Sparisoma viride. Biol. Let., 8: 282-286.

Chasqui V., L., A. Polanco F., A. Acero P., P.A. Mejía-Falla, A.F. Navia, L.A. Zapata y J.P. Caldas (Eds.). 2017. Libro rojo de peces marinos de Colombia. Serie Publicaciones Generales Invemar 93, Santa Marta. 552 p.

Dahl, G. 1964. Los peces cartilaginosos y del estuario de la bahía de Cispatá y del estuario del río Sinú. Rev. Acad. Colomb. Cienc., 12 (46): 175-195.

Dahl, G. 1971. Los peces del norte de Colombia. Inderena, Bogotá. $391 \mathrm{p}$.

Forbes, T., A. Rojas Archbold, A. Acero P., J.P. Caldas, E. Castro, L.O. Duarte y F. Gómez. 2017. Negaprion brevirostris (Poey, 1868): 278-280. En: Chasqui V., L., A. Polanco F., A. Acero P., P.A. Mejía-Falla, A.F. Navia, L.A. Zapata y J.P. Caldas (Eds.). 2017. Libro rojo de peces marinos de Colombia. Serie Publicaciones Generales Invemar 93, Santa Marta. 552 p.

Fowler, H.W. 1944. Results of the Catherwood-Chaplin West Indies Expedition, 1948. Part III. The fishes. Proc. Acad. Nat. Sci. Phila., 102: 69-93.

Fowler, H.W. 1953. The shore fishes of the Colombian Caribbean. Caldasia, 6 (27): 43-73.

Garzón, J. y A. Acero P. 1983. Notas sobre la pesca y los peces comerciales de la Isla de Providencia (Colombia), incluyendo nuevos registros para el Caribe occidental. Carib. J. Sci., 19 (3-4): 9-19.

Gómez, F., A. Acero P., J.P. Caldas, E. Castro y L.O. Duarte. 2017a. Carcharhinus perezii (Poey, 1876): 285-288. En: Chasqui V., L., A. Polanco F., A. Acero P., P.A. Mejía-Falla, A. F. Navia, L.A. Zapata y J.P. Caldas (Eds.). 2017. Libro rojo de peces marinos de Colombia. Serie Publicaciones Generales Invemar 93, Santa Marta. 552 p.

Gómez, F., A. Acero P., J.P. Caldas, E. Castro, L.O. Duarte y M. Amanza. 2017b. Rhizoprionodon porosus (Poey, 1861): 270-273. En: Chasqui V., L., A. Polanco F., A. Acero P., P.A. Mejía-Falla, A.F. Navia, L.A. Zapata y J.P. Caldas (Eds.). 2017. Libro rojo de peces marinos de Colombia. Serie Publicaciones Generales Invemar 93, Santa Marta. $552 \mathrm{p}$.

Gómez, F., A. Acero P., J.P. Caldas, E. Castro y L.O. Duarte. 2017c. Carcharhinus acronotus (Poey, 1860): 383-385. En: Chasqui V., L., A. Polanco F., A. Acero P., P.A. Mejía-Falla, A.F. Navia, L.A. Zapata y J.P. Caldas (Eds.). 2017. Libro rojo de peces marinos de Colombia. Serie Publicaciones Generales Invemar 93, Santa Marta. 552 p.

Gómez, F., A. Acero P., J.P. Caldas, E. Castro y L.O. Duarte. 2017d. Carcharhinus obscurus (Lesueur, 1818): 386388. En: Chasqui V., L., A. Polanco F., A. Acero P., P.A. Mejía-Falla, A.F. Navia, L.A. Zapata y J.P. Caldas (Eds.). 2017. Libro rojo de peces marinos de Colombia. Serie Publicaciones Generales Invemar 93, Santa Marta. 552 p.

Gómez, F., A. Acero P., J.P. Caldas, E. Castro y L.O. Duarte. 2017e. Carcharhinus porosus (Ranzani, 1839): 389391. En: Chasqui V., L., A. Polanco F., A. Acero P., P.A. Mejía-Falla, A.F. Navia, L.A. Zapata y J.P. Caldas (Eds.). 2017. Libro rojo de peces marinos de Colombia. Serie Publicaciones Generales Invemar 93, Santa Marta. 552 p.

Gómez-Rodríguez, S., J.P. Caldas, A. Acero P., M.A. MartínezSilva, P. Saénz-Okuyama, C.A. Lasso y O.M. LassoAlcalá. 2014. Geographic distribution and conservation 
status of sawfish Pristis spp. (Pristiformes: Pristidae) in the southern Caribbean sea. Biota Col., 15 (Supl. 1): 109-117.

González, J., M. Grijalba-Bendeck, A. Acero P. y R. Betancur-R. 2009. The invasive red lionfish, Pterois volitans (Linnaeus 1758), in the southwestern Caribbean Sea. Aq. Invas., 4 (3): 507-510.

González-Corredor, J.D., A. Acero P. y R. García-Urueña. 2016. Densidad y estructura de tallas del pez león Pterois volitans (Scorpaenidae) en el Caribe occidental insular colombiano. Bol. Invest. Mar. Cost., 45 (2): 317-333.

IUCN. 2017. The IUCN red list of threatened species. http://www. iucnredlist.org/

Knapp, L., M.M. Mincarone, H. Harwell, B. Polidoro, J. Sanciangco y K. Carpenter. 2011. Conservation status of world's hagfish species and the loss of phylogenetic diversity and ecosystem function. Aq. Conserv. Mar. Freshwater Ecosyst., 21: 401-411.

Last, P.R., B. Seret y G.J.P. Naylor. 2016a. A new species of guitarfish, Rhinobatos borneensis sp.nov. with a redefinition of the family-level classification in the order Rhinopristiformes (Chondrichthyes : Batoidea). Zootaxa, 4117 (4): 451-475.

Last, P.R., W.T. White, M.R. de Carvalho, B. Séret, M.F.W. Stehmann y G.J.P. Naylor (Eds.). 2016b. Rays of the world. Cornell, Ithaca, EE. UU. 790 p.

Linardich, C., G. Ralph, K. Carpenter, N. Cox, D.R. Robertson, H. Harwell, A. Acero P., K. Aiken, W. Anderson Jr., F. Barthelat, J. Bouchereau, J. Brown, J. Buchanan, D. Buddo, G. Bustamante, B. Collette, M. ComerosRaynal, M. Craig, M. Curtis, T. Defex, J. Dooley, C. Elfes Livsey, W. Eschmeyer, T. Fraser, R. Gilmore Jr., L. Grijalba Bendeck, A. Hines, R. Kishore, K. Lindeman, J. Marechal, J. McEachran, R. McManus, J. Moore, T. Munroe, H. Oxenford, F. Pezold, F. Pina Amargós, A. Polanco Fernández, B. Polidoro, C. Pollock, R. Robins, B. Russell, C. Sayer, S. Singh-Renton, W. Smith-Vaniz, L. Tornabene, J. Van Tassell, J.-C. Vié, J. Williams y B. Zane. 2017. The conservation status of marine bony shorefishes of the greater Caribbean. Gland, Switzerland: IUCN. viii +75 p.

Mace, G.M., N.J. Collar, K.J. Gaston, C. Hilton -Taylor, H.R. Akcakaya, N. Leader-Williams, E.J. Milner-Gulland y S.N. Stuart. 2008. Quantification of extinction risk: IUCN's system for classifying threatened species. Conserv. Bio., 22 (6): 1424-1442. DOI: 10.1111/j.1523-1739.2008.01044.x

Mejía-Falla, P.A. y A.F. Navia. 2017a. Pseudobatos prahli (Acero P. \& Franke 1995): 297-299. En: Chasqui V., L., A. Polanco F., A. Acero P., P.A. Mejía-Falla, A.F. Navia, L.A. Zapata y J.P. Caldas (Eds.). 2017. Libro rojo de peces marinos de Colombia. Serie Publicaciones Generales Invemar 93, Santa Marta. 552 p.

Mejía-Falla, P.A. y A.F. Navia. 2017b. Zapteryx xyster Jordan \& Evermann, 1896: 392-394. En: Chasqui V., L., A. Polanco F., A. Acero P., P.A. Mejía-Falla, A.F. Navia, L.A. Zapata y J.P. Caldas (Eds.). 2017. Libro rojo de peces marinos de Colombia. Serie Publicaciones Generales Invemar 93, Santa Marta. 552 p.

Mejía-Falla, P.A., A.F. Navia, S. Teillaud, L.H. Rodríguez, A. Suárez y L.A. Zapata. 2017a. Pseudobatos leucorhynchus (Günther, 1867): 148-151. En: Chasqui V., L., A. Polanco F., A. Acero P., P.A. Mejía-Falla, A.F. Navia, L.A. Zapata y J.P. Caldas (Eds.). 2017. Libro rojo de peces marinos de Colombia. Serie Publicaciones Generales Invemar 93, Santa Marta. 552 p.

Mok, H.K., L. Saavedra y A. Acero P. 2001. Two new species of Eptatretus and Quadratus (Myxinidae: Myxiniformes) from the Caribbean coast of Colombia. Copeia, 2001 (4): 1026-1033.

Navia, A.F., P.A. Mejía-Falla y J.S. Hleap. 2016. Zoogeography of elasmobranchs in the Colombian Pacific Ocean and Caribbean Sea. Neotrop. Ichthyol., 14(2): e140134. Epub July 07, 2016.https://dx.doi.org/10.1590/1982-0224-20140134

Navia, A.F., P.A. Mejía-Falla, S. Teillaud, M.C. Diazgranados, A. Suárez, V. Puentes, L.A. Zapata, F. Gómez, A. Acero P., J.P. Caldas, E. Castro y L.O. Duarte. 2017. Carcharhinus limbatus Müller y Henle, 1839: 126-130. En: Chasqui V., L., A. Polanco F., A. Acero P., P.A. Mejía-Falla, A.F. Navia, L.A. Zapata y J.P. Caldas (Eds.). 2017. Libro rojo de peces marinos de Colombia. Serie Publicaciones Generales Invemar 93, Santa Marta. 552 p.

Neira, A. y A. Acero P. 2016. Megalops atlanticus (Megalopidae), un nuevo pez en el océano Pacífico; información sobre su importancia pesquera. Rev. MVZ Córdoba, 21 (3): 55255534.

Nelson, J. S., T.C. Grande y M.V.H. Wilson. 2016. Fishes of the world. Quinta edición. Wiley, New Jersey. 707 p.

Palacio, F. J. 1974. Peces colectados en el Caribe colombiano por la Universidad de Miami. Bol. Mus. Mar, 6: 116 p.

Payán, L.F., A.F. Navia, E.A. Rubio y P.A. Mejía-Falla. 2011. Biología de la raya guitarra Rhinobatos leucorhynchus en el Pacífico colombiano. Latin Am. J. Aq. Res., 39 (2): 286-296.

Polanco Fernández, A. 2015. Dynamics of the continental slope demersal fish community in the Colombian Caribbean Deep sea research in the Caribbean. Dis. Doct. Interinst. Cienc. Mar, Univ. Nal. Col., Santa Marta.

Polanco F., A. y A. Acero P. 2017a. Hyporthodus nigritus (Holbrook, 1855): 440-442. En: Chasqui V., L., A. Polanco F., A. Acero P., P.A. Mejía-Falla, A.F. Navia, L.A. Zapata y J.P. Caldas (Eds.). 2017. Libro rojo de peces marinos de Colombia. Serie Publicaciones Generales Invemar 93, Santa Marta. 552 p.

Polanco F., A. y A. Acero P. 2017b. Hypoplectrus providencianus Acero P. \& Garzón-Ferreira, 1994: 341-343. En: Chasqui V., L., A. Polanco F., A. Acero P., P.A. Mejía-Falla, A.F. Navia, L.A. Zapata y J.P. Caldas (Eds.). 2017. Libro rojo de peces marinos de Colombia. Serie Publicaciones Generales Invemar 93, Santa Marta. 552 p.

Polanco Fernández, A. y B. Ferholm. 2014. A new species of hagfish (Myxinidae: Eptatetrus) from the Colombian Caribbean. Copeia, 2017 (3): 530-533.

Polanco F., A., A. Acero P. y M. Almanza. 2017. Epinephelus itajara: 61-64. En: Chasqui V., L., A. Polanco F., A. Acero P., P.A. Mejía-Falla, A.F. Navia, L.A. Zapata y J.P. Caldas (Eds.). 2017. Libro rojo de peces marinos de Colombia. Serie Publicaciones Generales Invemar 93, Santa Marta. $552 \mathrm{p}$.

Rey Carrasco, I. y A. Acero P. 2002. Biodiversidad íctica del Caribe colombiano. Univ. Jorge Tadepo Lozano, Bogotá. 190 p. http://avalon.utadeo.edu.co/dependencias/publicaciones/ pelect_biologia.php.

Rubio, E. A., B. Gutiérrez y R. Franke. 1987. Peces de la isla Gorgona. Univ. Valle, Cali. 315 p. 
Rubio, E.A., M.J. Pedraza, J. Milton y L.A. Zapata. 2005. Primer hallazgo de Myxine circifrons Garman 1899 (AGNATHA: MYXINIDAE) en la costa del Pacífico de Colombia. Gayana (Concepción), 69 (1): 118-121.

van der Laan, R., W. N. Eschmeyer and R. Fricke. Family-group names (http://www.calacademy.org/scientists/catalog-offishes-family-group-names/).

Westneat, M.W. y M.E. Alfaro. 2005. Phylogenetic relationship and evolutionary history of the reef fish family Labridae. Mol. Phylogen. Evol., 36: 370-390.

Wisner, R.L. y C.B. McMillan. 1995. Review of new world hagfishes of the genus Myxine (Agnata:Myxinidae) with descriptions of nine new species. Fish. Bull., 93 (3): 530550 .
Zapata, F. y L. Chasqui V. 2017. Halichoeres malpelo Allen \& Robertson, 1992: 219-221. En: Chasqui V., L., A. Polanco F., A. Acero P., P.A. Mejía-Falla, A.F. Navia, L.A. Zapata y J.P. Caldas (Eds.). 2017. Libro rojo de peces marinos de Colombia. Serie Publicaciones Generales Invemar 93, Santa Marta. 552 p.

Zapata, L.A., A.F. Navia, J.P. Caldas, P.A. Mejía-Falla, J. Botero, S. Teillaud, M.C. Diazgranados, A. Suárez, V. Puentes y C.J. Polo Silva. 2017. Carcharhinus falciformis Müller y Henle, 1839: 121-125. En: Chasqui V., L., A. Polanco F., A. Acero P., P.A. Mejía-Falla, A.F. Navia, L.A. Zapata y J.P. Caldas (Eds.). Libro rojo de peces marinos de Colombia. Serie Publicaciones Generales Invemar 93, Santa Marta. 552 p.

\section{Anexo}

Lista filogenética de los peces marinos amenazados de Colombia. De cada especie se indican la distribución en Colombia (C Caribe, P Pacífico, CP ambos mares), la categoría de amenaza (CR críticamente amenazada, EN en peligro, VU vulnerable, NT casi amenazada y DD datos insuficientes) y, para las especies incluidas en el Libro Rojo, los factores de amenaza detectados (SP sobrepesca, DH destrucción del hábitat, HV historia de vida, ENSO El Niño Oscilación del Sur, CO colisiones) según los autores de cada ficha en el Libro Rojo (Chasqui V., et al., 2017). Las especies colombianas categorizadas como amenazadas por la IUCN, pero no incluidas en la segunda edición del Libro Rojo colombiano se indican con un asterisco (*).

\begin{tabular}{|c|c|c|c|}
\hline Taxa & Distribución & Categoría & Amenazas \\
\hline \multicolumn{4}{|l|}{ Clase Chondrichthyes } \\
\hline \multicolumn{4}{|l|}{ Orden Orectolobiformes } \\
\hline \multicolumn{4}{|l|}{ Familia Rhincodontidae } \\
\hline Rhincodon typus Smith, 1828 & $\mathrm{CP}$ & DD & $\mathrm{CO}$ \\
\hline \multicolumn{4}{|l|}{ Familia Ginglymostomidae } \\
\hline Ginglymostoma cirratum (Bonnaterre, 1788) & $\mathrm{C}$ & VU & SP, DH, HV \\
\hline \multicolumn{4}{|l|}{ Orden Lamniformes } \\
\hline \multicolumn{4}{|l|}{ Familia Odontaspididae } \\
\hline Odontaspis ferox (Risso, 1810)* & $\mathrm{P}$ & VU & \\
\hline \multicolumn{4}{|l|}{ Familia Lamnidae } \\
\hline Isurus oxyrinchus Rafinesque, 1810 & $\mathrm{C}$ & DD & SP \\
\hline Isurus paucus Guitart Manday, 1966* & $\mathrm{C}$ & VU & \\
\hline \multicolumn{4}{|l|}{ Familia Alopiidae } \\
\hline Alopias pelagicus Nakamura, 1935 & $\mathrm{P}$ & VU & SP \\
\hline Alopias superciliosus Lowe, 1841 & $\mathrm{CP}$ & NT & SP \\
\hline \multicolumn{4}{|l|}{ Orden Carcharhiniformes } \\
\hline \multicolumn{4}{|l|}{ Familia Triakidae } \\
\hline Mustelus henlei (Gill, 1863) & $\mathrm{P}$ & VU & SP \\
\hline Mustelus lunulatus Jordan y Gilbert, 1882 & $\mathrm{P}$ & VU & SP \\
\hline Mustelus minicanis Heemstra, 1997 & $\mathrm{C}$ & VU & SP \\
\hline \multicolumn{4}{|l|}{ Familia Carcharhinidae } \\
\hline Carcharhinus acronotus (Poey, 1860) & $\mathrm{C}$ & DD & SP, DH \\
\hline Carcharhinus falciformis (Müller y Henle, 1839) & $\mathrm{CP}$ & VU & SP \\
\hline Carcharhinus limbatus (Müller y Henle, 1839) & $\mathrm{CP}$ & VU & SP, DH, HV \\
\hline Carcharhinus longimanus (Poey, 1861) & $\mathrm{CP}$ & VU & SP \\
\hline Carcharhinus obscurus (Lesueur, 1818) & $\mathrm{C}$ & DD & SP, DH \\
\hline Carcharhinus perezii (Poey, 1876) & $\mathrm{C}$ & NT & SP \\
\hline Carcharhinus porosus (Ranzani, 1839) & $\mathrm{C}$ & DD & SP, DH \\
\hline
\end{tabular}




\begin{tabular}{|c|c|c|c|}
\hline Taxa & Distribución & Categoría & Amenazas \\
\hline Carcharhinus signatus (Poey, 1868)* & $\mathrm{C}$ & VU & \\
\hline Galeocerdo cuvier (Péron y Lesueur, 1822) & $\mathrm{CP}$ & NT & SP, HV \\
\hline Negaprion brevirostris (Poey, 1868) & $\mathrm{CP}$ & NT & SP, HV \\
\hline Prionace glauca (Linnaeus, 1758) & $\mathrm{CP}$ & NT & SP \\
\hline Rhizoprionodon porosus (Poey, 1861) & $\mathrm{C}$ & NT & SP \\
\hline \multicolumn{4}{|l|}{ Familia Sphyrnidae } \\
\hline Sphyrna corona Springer, 1940 & $\mathrm{P}$ & NT & SP \\
\hline Sphyrna lewini (Griffith y Smith, 1834) & $\mathrm{CP}$ & VU & SP, HV \\
\hline Sphyrna mokarran (Ruppell, 1837) & $\mathrm{CP}$ & VU & SP, HV \\
\hline Sphyrna tudes (Valenciennes, 1822)* & $\mathrm{C}$ & VU & \\
\hline \multicolumn{4}{|l|}{ Orden Torpediniformes } \\
\hline \multicolumn{4}{|l|}{ Familia Narcinidae } \\
\hline Diplobatis colombiensis Fechhelm y McEachran, 1984 & $\mathrm{C}$ & EN & SP \\
\hline Diplobatis guamachensis Martín Salazar, 1957 & $\mathrm{C}$ & VU & SP \\
\hline Diplobatis ommata (Jordan y Gilbert, 1890)* & $P$ & VU & \\
\hline Narcine bancroftii (Griffith y Smith, 1834)* & $\mathrm{C}$ & CR & \\
\hline Narcine leoparda Carvalho, 2001 & $\mathrm{P}$ & NT & SP, DH \\
\hline \multicolumn{4}{|l|}{ Orden Rhinopristiformes } \\
\hline \multicolumn{4}{|l|}{ Familia Trygonorrhinidae } \\
\hline Zapteryx xyster Jordan y Evermann, 1896 & $\mathrm{P}$ & DD & SP \\
\hline \multicolumn{4}{|l|}{ Familia Rhinobatidae } \\
\hline Pseudobatos leucorhynchus (Günther, 1867) & $\mathrm{P}$ & VU & SP, DH \\
\hline Pseudobatos prahli (Acero P. y Franke, 1995) & $\mathrm{P}$ & NT & SP, DH \\
\hline \multicolumn{4}{|l|}{ Familia Pristidae } \\
\hline Pristis pectinata Latham, 1794 & $\mathrm{C}$ & CR & SP, DH, HV \\
\hline Pristis pristis (Linnaeus, 1758) & $\mathrm{CP}$ & CR & SP, HV \\
\hline \multicolumn{4}{|l|}{ Orden Myliobatiformes } \\
\hline \multicolumn{4}{|l|}{ Familia Dasyatidae } \\
\hline Hypanus americanus (Hildebrand y Schroeder, 1928) & $\mathrm{C}$ & NT & SP, DH \\
\hline Hypanus longus (Garman, 1880) & $\mathrm{P}$ & VU & SP \\
\hline \multicolumn{4}{|l|}{ Familia Aetobatidae } \\
\hline Aetobatus narinari (Euphrasen, 1790) & $\mathrm{C}$ & NT & SP, DH, HV \\
\hline \multicolumn{4}{|l|}{ Familia Rhinopteridae } \\
\hline Rhinoptera brasiliensis Müller, 1836* & $\mathrm{C}$ & EN & \\
\hline \multicolumn{4}{|l|}{ Familia Mobulidae } \\
\hline Manta birostris (Walbaum, 1792) & $\mathrm{CP}$ & DD & SP, HV \\
\hline \multicolumn{4}{|l|}{ Clase Actinopteri } \\
\hline \multicolumn{4}{|l|}{ Orden Elopiformes } \\
\hline \multicolumn{4}{|l|}{ Familia Megalopidae } \\
\hline Megalops atlanticus Valenciennes, 1847 & $\mathrm{CP}$ & CR & SP, DH, HV \\
\hline \multicolumn{4}{|l|}{ Orden Clupeiformes } \\
\hline \multicolumn{4}{|l|}{ Familia Engraulidae } \\
\hline Cetengraulis edentulus (Cuvier, 1829) & $\mathrm{C}$ & NT & SP, DH \\
\hline \multicolumn{4}{|l|}{ Orden Siluriformes } \\
\hline \multicolumn{4}{|l|}{ Familia Ariidae } \\
\hline Ariopsis canteri Acero P., Betancur-R. y Marceniuk, 2017 & $\mathrm{C}$ & EN & SP, DH \\
\hline Cathorops mapale Betancur-R. y Acero P., 2005 & $\mathrm{C}$ & VU & SP, DH \\
\hline Sciades proops (Valenciennes, 1840) & $\mathrm{C}$ & VU & SP, DH \\
\hline
\end{tabular}




\begin{tabular}{|c|c|c|c|}
\hline Taxa & Distribución & Categoría & Amenazas \\
\hline \multicolumn{4}{|l|}{ Orden Ophidiiformes } \\
\hline \multicolumn{4}{|l|}{ Familia Bythitidae } \\
\hline Ogilbichthys ferocis Møller, Schwarzhans y Nielsen, 2004* & $\mathrm{C}$ & VU & \\
\hline Parasaccogaster melanomycter (Cohen, 1981) & $\mathrm{C}$ & DD & DH \\
\hline \multicolumn{4}{|l|}{ Familia Ophidiidae } \\
\hline Brotula clarkae Hubbs, 1944 & $\mathrm{P}$ & NT & SP \\
\hline \multicolumn{4}{|l|}{ Orden Batrachoidiformes } \\
\hline \multicolumn{4}{|l|}{ Familia Batrachoididae } \\
\hline Batrachoides manglae Cervigón, 1964 & $\mathrm{C}$ & VU & DH \\
\hline \multicolumn{4}{|l|}{ Orden Scombriformes } \\
\hline \multicolumn{4}{|l|}{ Familia Pomatomidae } \\
\hline Pomatomus saltatrix (Linnaeus, 1766)* & $\mathrm{C}$ & VU & \\
\hline \multicolumn{4}{|l|}{ Familia Scombridae } \\
\hline Katsuwonus pelamis (Linnaeus, 1758) & $\mathrm{CP}$ & DD & SP \\
\hline Scomberomorus sierra Jordan y Starks, 1895 & $\mathrm{P}$ & NT & SP \\
\hline Thunnus alalunga (Bonnaterre, 1788) & $\mathrm{C}$ & DD & SP \\
\hline Thunnus albacares (Bonnaterre, 1788) & $\mathrm{CP}$ & NT & SP \\
\hline Thunnus obesus (Lowe, 1839) & $\mathrm{CP}$ & VU & SP \\
\hline
\end{tabular}

\section{Orden Syngnathiformes}

Familia Syngnathidae

Hippocampus erectus Perry, 1810

$\begin{array}{lll}\text { C } & \text { VU } & \text { SP, DH } \\ \text { P } & \text { VU } & \text { SP, DH } \\ \text { C } & \text { VU } & \text { SP, DH }\end{array}$

Hippocampus ingens Girard, 1858

Hippocampus reidi Ginsburg, 1939

Orden Gobiiformes

Familia Gobiidae

Coryphopterus alloides Böhlke y Robins, 1960*

Coryphopterus eidolon Böhlke y Robins, 1960*

Coryphopterus hyalinus Böhlke y Robins, 1960*

Coryphopterus lipernes Böhlke y Robins, 1960*

Coryphopterus personatus (Jordan y Thompson, 1905)*

Coryphopterus thrix Böhlke y Robins, 1960*

Coryphopterus tortugae (Jordan, 1904)*

Coryphopterus venezuelae Cervigón, 1966*

Chriolepis lepidota Findley, 1975

Elacatinus prochilos (Böhlke y Robins, 1968)*

Gobiosoma spilotum (Ginsburg, 1939)*

Priolepis robinsi Garzón-Ferreira y Acero P., 1991

Tigrigobius nesiotes (Bussing, 1990)

Carangaria (orden incertae sedis)

Familia Centropomidae

Centropomus armatus Gill, 1863

Centropomus undecimalis (Bloch, 1792)

Familia Sphyraenidae

Sphyraena barracuda (Edwards, 1771)

Orden Istiophoriformes

Familia Istiophoridae

Makaira nigricans Lacepède, 1802

C VU

C VU

C VU

C VU

C VU

C VU

C VU

C VU

$\mathrm{P}$ DD

C VU

C VU

C DD

$\mathrm{P} \quad \mathrm{VU}$

$\mathrm{DH}$

VU ENSO

Familia Xiphiidae

Xiphias gladius Linnaeus, 1758

$\mathrm{P}$

C

C

CP

CP

DD

SP

VU SP, HV

NT $\quad$ SP, DH




\begin{tabular}{|c|c|c|c|}
\hline Taxa & Distribución & Categoría & Amenazas \\
\hline \multicolumn{4}{|l|}{ Orden Carangiformes } \\
\hline \multicolumn{4}{|l|}{ Familia Carangidae } \\
\hline Caranx hippos (Linnaeus, 1766) & $\mathrm{C}$ & VU & SP, HV \\
\hline \multicolumn{4}{|l|}{ Ovalentaria (orden incertae sedis) } \\
\hline \multicolumn{4}{|l|}{ Familia Pomacentridae } \\
\hline Stegastes beebei (Nichols, 1924) & $\mathrm{P}$ & VU & ENOS \\
\hline \multicolumn{4}{|l|}{ Orden Beloniformes } \\
\hline \multicolumn{4}{|l|}{ Familia Belonidae } \\
\hline Tylosurus pacificus (Steindachner, 1876) & $\mathrm{P}$ & DD & SP \\
\hline \multicolumn{4}{|l|}{ Orden Cyprinodontiformes } \\
\hline \multicolumn{4}{|l|}{ Familia Poeciliidae } \\
\hline Gambusia aestiputeus Fowler, 1950 & $\mathrm{C}$ & VU & DH \\
\hline Gambusia lemaitrei Fowler, 1950 & $\mathrm{C}$ & CR & DH \\
\hline \multicolumn{4}{|l|}{ Orden Mugiliformes } \\
\hline \multicolumn{4}{|l|}{ Familia Mugilidae } \\
\hline Joturus pichardi Poey, 1860 & $\mathrm{C}$ & VU & SP, DH, HV \\
\hline Mugil incilis Hancock, 1830 & $\mathrm{C}$ & VU & SP, DH \\
\hline Mugil liza Valenciennes, 1836 & $\mathrm{C}$ & VU & SP, HV \\
\hline \multicolumn{4}{|l|}{ Orden Blenniiformes } \\
\hline \multicolumn{4}{|l|}{ Familia Chaenopsidae } \\
\hline Acanthemblemaria stephensi Rosenblatt y McCosker, 1988 & $\mathrm{P}$ & VU & ENOS \\
\hline Emblemariopsis tayrona (Acero P., 1987) & $\mathrm{C}$ & DD & $\mathrm{DH}$ \\
\hline \multicolumn{4}{|l|}{ Familia Tripterygiidae } \\
\hline Axoclinus rubinoffi Allen y Robertson, 1992 & $\mathrm{P}$ & VU & ENOS \\
\hline Lepidonectes bimaculatus Allen y Robertson, 1992 & $\mathrm{P}$ & VU & ENOS \\
\hline \multicolumn{4}{|l|}{ Eupercaria (orden incertae sedis) } \\
\hline \multicolumn{4}{|l|}{ Familia Sciaenidae } \\
\hline Cynoscion phoxocephalus Jordan y Gilbert, 1882 & $\mathrm{P}$ & VU & SP \\
\hline \multicolumn{4}{|l|}{ Orden Gerreiformes } \\
\hline \multicolumn{4}{|l|}{ Familia Gerreidae } \\
\hline Eugerres plumieri (Cuvier, 1830) & $\mathrm{C}$ & VU & DH \\
\hline \multicolumn{4}{|l|}{ Orden Labriformes } \\
\hline \multicolumn{4}{|l|}{ Familia Labridae } \\
\hline Halichoeres malpelo Allen y Robertson, 1992 & $\mathrm{P}$ & VU & ENOS \\
\hline Lachnolaimus maximus (Walbaum, 1792) & $\mathrm{C}$ & EN & SP, DH, HV \\
\hline Scarus coelestinus Valenciennes, 1840 & C & EN & SP, DH \\
\hline Scarus coeruleus (Edwards, 1771) & $\mathrm{C}$ & EN & SP, DH, HV \\
\hline Scarus guacamaia Cuvier, 1829 & $\mathrm{C}$ & EN & SP, DH, HV \\
\hline Scarus vetula Bloch y Schneider, 1801 & $\mathrm{C}$ & NT & SP, DH \\
\hline Sparisoma viride (Bonnaterre, 1788) & $\mathrm{C}$ & NT & SP, DH \\
\hline \multicolumn{4}{|l|}{ Orden Lutjaniformes } \\
\hline \multicolumn{4}{|l|}{ Familia Haemulidae } \\
\hline Anisotremus moricandi (Ranzani, 1842) & $\mathrm{C}$ & VU & DH \\
\hline \multicolumn{4}{|l|}{ Familia Lutjanidae } \\
\hline Lutjanus analis (Cuvier, 1828) & $\mathrm{C}$ & VU & SP, DH \\
\hline Lutjanus campechanus (Poey, 1860)* & $\mathrm{C}$ & VU & \\
\hline Lutjanus cyanopterus (Cuvier, 1828) & $\mathrm{C}$ & VU & SP, DH, HV \\
\hline Lutjanus guttatus (Steindachner, 1869) & $\mathrm{P}$ & NT & SP \\
\hline Lutjanus jocu (Bloch y Schneider, 1801) & $\mathrm{C}$ & DD & SP \\
\hline
\end{tabular}




\begin{tabular}{|c|c|c|c|}
\hline Taxa & Distribución & Categoría & Amenazas \\
\hline Lutjanus peru (Nichols y Murphy, 1922) & $\mathrm{P}$ & NT & SP \\
\hline Ocyurus chrysurus (Bloch, 1791) & $\mathrm{C}$ & NT & SP \\
\hline Rhomboplites aurorubens (Cuvier, 1829)* & $\mathrm{C}$ & VU & \\
\hline \multicolumn{4}{|l|}{ Orden Spariformes } \\
\hline \multicolumn{4}{|l|}{ Familia Sparidae } \\
\hline Pagrus pagrus (Linnaeus, 1758) & $\mathrm{C}$ & DD & SP \\
\hline \multicolumn{4}{|l|}{ Orden Tetraodontiformes } \\
\hline \multicolumn{4}{|l|}{ Familia Molidae } \\
\hline Mola mola (Linnaeus, 1758)* & $\mathrm{CP}$ & VU & \\
\hline \multicolumn{4}{|l|}{ Familia Balistidae } \\
\hline Balistes capriscus Gmelin, 1789* & $\mathrm{C}$ & VU & \\
\hline Balistes vetula Linnaeus, 1758 & $\mathrm{C}$ & EN & SP \\
\hline \multicolumn{4}{|l|}{ Familia Tetraodontidae } \\
\hline Sphoeroides georgemilleri Shipp, 1972 & $\mathrm{C}$ & DD & SP \\
\hline \multicolumn{4}{|l|}{ Orden Perciformes } \\
\hline \multicolumn{4}{|l|}{ Familia Serranidae } \\
\hline Dermatolepis inermis (Valenciennes, 1833) & $\mathrm{C}$ & DD & DH \\
\hline Epinephelus cifuentesi Lavenberg y Grove, 1993 & $\mathrm{P}$ & VU & SP \\
\hline Epinephelus guttatus (Linnaeus, 1758) & $\mathrm{C}$ & NT & SP, DH \\
\hline Epinephelus itajara (Lichtenstein, 1822) & $\mathrm{C}$ & CR & SP, DH, HV \\
\hline Epinephelus quinquefasciatus (Bocourt, 1868) & $\mathrm{P}$ & DD & SP \\
\hline Epinephelus striatus (Bloch, 1792) & $\mathrm{C}$ & CR & $\mathrm{SP}, \mathrm{DH}$ \\
\hline Hypoplectrus providencianus Acero P. y Garzón-Ferreira, 1994 & $\mathrm{C}$ & NT & $\mathrm{DH}$ \\
\hline Hyporthodus acanthistius (Gilbert, 1892) & $\mathrm{P}$ & NT & SP \\
\hline Hyporthodus nigritus (Holbrook, 1855) & $\mathrm{C}$ & $\mathrm{DD}$ & $\mathrm{DH}$ \\
\hline Hyporthodus niphobles (Gilbert y Starks, 1897) & $\mathrm{P}$ & DD & SP \\
\hline Hyporthodus niveatus (Valenciennes, 1828) & $\mathrm{C}$ & DD & SP \\
\hline Mycteroperca bonaci (Poey, 1860) & $\mathrm{C}$ & VU & SP, DH, HV \\
\hline Mycteroperca cidi Cervigón, 1966 & $\mathrm{C}$ & VU & SP \\
\hline Mycteroperca interstitialis (Poey, 1860)* & $\mathrm{C}$ & VU & \\
\hline Mycteroperca olfax (Jenyns, 1840) & $\mathrm{P}$ & VU & SP \\
\hline Mycteroperca tigris (Valenciennes, 1833) & $\mathrm{C}$ & NT & SP, DH \\
\hline Mycteroperca venenosa (Linnaeus, 1758) & $\mathrm{C}$ & VU & SP, DH \\
\hline Mycteroperca xenarcha Jordan, 1888 & $\mathrm{P}$ & DD & SP \\
\hline
\end{tabular}

